\title{
Marie Joseph Chénier : de la pratique de la traduction à l'introduction d'œuvres traduites dans le canon littéraire
}

\author{
Jean-Jacques Tatin-Gourier \\ Université François Rabelais, Tours
}

La postérité a certes retenu le nom de Chénier, mais il s'agit en fait d'André, le poète emprisonné et exécuté sous la Terreur. Cependant pour les contemporains et jusqu'aux années 1820 , le nom de Chénier renvoyait d'abord à Marie Joseph (1764-1811) indissociablement dramaturge, poète, homme politique et critique littéraire. C'est en 1789, peu après la prise de la Bastille que Marie Joseph Chénier dramaturge connut un succès retentissant avec sa pièce Charles IX ou la Saint-Barthélemy. Les pièces qui suivirent au long des années révolutionnaires et sous le Consulat (Henri VIII et Jean Calas, 1791; Cä̈us Gracchus, 1792; Fénelon, 1793; Timoléon, 1794, Cyrus, 1804) traitèrent des mêmes thèmes (l'exercice du pouvoir, le fanatisme et la liberté) mais connurent des succès inégaux et déclenchèrent parfois des polémiques liées à une carrière politique complexe et à un engagement constant notamment marqué par l'écriture d'hymnes patriotiques ${ }^{1}$ et par le rôle d'organisateur des grandes fêtes révolutionnaires aux côtés du peintre David et du compositeur Gossec.

L’itinéraire politique de Marie Joseph Chénier fut intégralement marqué par le développement du processus révolutionnaire. Dantoniste, membre du Club des Cordeliers et de la Commune de Paris, député à la Convention, Marie Joseph Chénier vota la mort de Louis XVI. Surveillé et menacé par Robespierre peu avant la chute de ce dernier, il ne put sauver son frère André de la guillotine. Thermidor et le Directoire lui permirent de reprendre son activité de dramaturge, de poète et peut-être surtout de critique littéraire, gardien vigilant du legs philosophique et politique des Lumières et de la Révolution, dénonçant sans trêve les écrivains 'réactionnaires'. La Harpe, Mme de Genlis et un peu plus tard, Chateaubriand, auteur en 1802 du Génie du christianisme, furent particulièrement pris pour cibles. Opposant républicain sous l'Empire (non sans ambiguïtés et sans ralliements apparents), Marie Joseph Chénier académicien fut remplacé à sa mort par Chateaubriand. ${ }^{2}$

Nombreux sont les historiens et les historiens de la littérature qui développent leur recherche sur la période qui embrasse la fin de la dynamique révolutionnaire, le consulat et l'empire. Et dans le cadre de ces questionnements, la figure de Marie Joseph Chénier ne peut que ressurgir et peut-être même s'avérer incontournable (Baczko ; Tatin-Gourier ; Walton). Face aux innovations thématiques et formelles des écrivains qu'il dénonce comme "nouveaux saints" (au rang desquels Chateaubriand est évidemment une cible majeure), face à certaines influences poétiques et romanesques nordiques et notamment allemandes, Marie Joseph Chénier se montre le gardien sourcilleux

\footnotetext{
${ }^{1}$ Le Chant du départ demeure un chant national connu (Domine).

${ }^{2}$ Voir à ce sujet le chapitre « Mort de Chénier » dans les Mémoires d'outre-tombe (I, 644$660)$.
} 
de l'héritage classique. Mais ce souci de préservation inclut aussi l'héritage philosophique et littéraire particulièrement mis à mal par la première grande histoire littéraire conçue par La Harpe (le Cours complet de littérature ou Lycée) et par le Génie du christianisme de Chateaubriand qui propose non seulement un canon littéraire nouveau foncièrement hostile au 'Goliath voltairien', mais une véritable idéologie de substitution marquée par le retour aux sources chrétiennes de la civilisation européenne.

Sur cette question de la défense du canon littéraire hérité des Lumières et de la Révolution, par delà les nombreuses épigrammes dans lesquelles Marie Joseph Chénier dénonce inlassablement les écrivains 'réactionnaires', l'ouvrage le plus révélateur est sans doute le Tableau historique de l'état et des progrès de la littérature française depuis 1789, ouvrage posthume publié en 1816 et revu ensuite sur les manuscrits dans les éditions suivantes. Aux côtés de deux éléments paratextuels antérieurs, c'est précisément la lecture de cet ouvrage divisé en grandes rubriques concernant les genres littéraires qui nous permettra d'apprécier la place que Marie Joseph Chénier assigne aux œuvres traduites en français dans le canon littéraire et philosophique qu'il élabore dans une dynamique certes avant tout défensive mais qui présente cependant un degré d'ouverture aux transferts culturels et littéraires qu'il importe de mesurer.

Mais il faut tout d'abord rappeler que Marie Joseph Chénier a été luimême traducteur et qu'il s'est aussi montré un critique attentif aux traductions publiées de son temps. Avant la Révolution, le jeune Marie Joseph Chénier a tout d'abord traduit ou "imité" certains fragments de grands poèmes latins. Les éditions posthumes dites complètes reprendront notamment la "traduction libre" du début du poème de Lucrèce, les "imitations" en vers d'un "morceau" des Géorgiques et du quatrième livre de l'Enéide. En 1818, l'édition de L.G.Michaud, destinée à l'usage des classes, accordera une place importante à deux traductions intégrales : l'Art poétique d'Horace, en vers, avec le texte latin en regard, précédé de la Poétique d'Aristote, en prose, avec pour elle aussi, le texte en regard. Il est à noter que les éditions suivantes, dites complètes, ne conserveront plus cette double disposition pour le texte d'Aristote. L'édition de 1824 des œuvres posthumes révèle aussi la traduction du Dialogue des orateurs attribué à Quintilien. On remarque également cinq Chants imités d'Ossian, traductions très libres des poèmes de Macpherson, publiés en l'an V de la République, ce qui démontre une ouverture aux œuvres poétiques contemporaines. De la même façon, Chénier-dramaturge s'est inspiré ou a imité des œuvres anciennes mais aussi contemporaines. Il a ainsi produit un EdipeRoi et un Edipe à Colone, il est allé puiser dans le Jules César de Shakespeare pour son Brutus et Cassius, dans le Dom Carlos de Schiller pour son Philippe II et a livré un «Nathan le Sage. Drame en trois actes et en vers imité de l'allemand de Schiller ». Nous n'avons retrouvé que trois textes dans lesquels Chénier expose sa conception de la traduction et où se dessinent les contours de la figure de traducteur. Tout d'abord une note, fournie par Daunou, sur l'EEdipe à Colone où Chénier indique qu'il faudrait selon lui le distinguer des traductions : 
La tragédie d'Edipe mourant n'est point une traduction de la pièce de Sophocle, mais une pièce sur le même sujet, où j'ai tâché de rassembler, autant qu'il m'a été possible, toutes les beautés de CEdipe à Colone, et de ne point le déshonorer par une intrigue oiseuse ou mesquine. J'ai retranché ce qui m'a semblé ne pouvoir convenir au théâtre français; mais, dans ce que j'ai ajouté, mon unique dessein a été d'imiter la manière dont ces Grecs pensaient, et exprimaient leurs pensées. Aucune nation ne les a égalés dans le style noble et simple, qui n'est point le style prosaïque, niais bien le style de la plus belle poésie, puisqu'il consiste à peindre toujours par l'expression et par les sons, sans jamais rien affecter. (Oeuvres posthumes I, vi)

Admettant ajouts et retranchements, Chénier revendique l'imitation, ce qui semble indiquer qu'il destinait cette pièce à la scène et non à des fins éducatives, suivant en cela une distinction entre imitation et traduction remarquée à l'époque (Lechevalier et Marie 918). Cette attitude transparaît dans le titre même de son Nathan le Sage, et en même temps se double d'une visée idéologique plus marquée que dans l'original (Ferry et Humbert-Mougin 472474).

Ensuite, la préface à sa traduction de l'élégie de Thomas Gray, Le Cimetière de campagne, publiée en 1805, s'avère riche d'enseignements. Notons d'emblée que nous retrouvons dans ce texte le caractère bilingue de la publication par la juxtaposition du texte original et du texte traduit. Le traducteur justifie le caractère bilingue de la publication par l'utilité d'une sorte d'exhibition d'un travail de traduction qui demeure selon lui provisoire et qui peut être amélioré par les lecteurs, traducteurs potentiels :

En donnant au public cette version nouvelle, composée il y a plusieurs années, je fais imprimer les vers anglais à côté des vers français. On pourra voir d'un coup d'œil ce que j'ai cru devoir supprimer, changer, ajouter; on jugera si j'ai su garder un juste milieu entre une imitation infidèle et une traduction servile. J'ai craint pour l'élégie entière la monotonie des stances; j'ai conservé seulement dans l'épitaphe ces formes de poésie qui m'ont paru lui convenir. J'ai travaillé cette pièce avec soin; mais, en quelque genre que ce soit, je n'ai jamais donné mes écrits que comme des essais susceptibles d'un perfectionnement graduel. Je serai disposé dans tous les temps à mettre à profit l'opinion des connaisseurs, et même ce que pourront offrir de judicieux les critiques amères des censeurs de profession. (Le cimetière iii-iv)

De plus, en un mouvement inverse, Marie Joseph Chénier présente sa « nouvelle traduction » dans le prolongement de traductions imparfaites du passé qu'il considère comme " plutôt des paraphrases que des traductions " (iii) sur lesquelles il intervient pour les corriger et les parfaire. ${ }^{3}$ Il inscrit d'ailleurs son intervention dans une dynamique historique séculaire de découverte, de traduction et d'appropriation de la littérature anglaise, dynamique initiée par Voltaire, par Ducis adaptateur du théâtre de Shakespeare et traducteur du Paradis perdu de Milton, puis développée par Delille traducteur de l'Epitre de Pope (iv). Et Marie Joseph Chénier, selon un usage assez répandu chez ses

${ }^{3}$ Sur les multiples traductions de l'Elégie à l'articulation des XVIIIe et XIX siècles, voir LautelRibstein, «Poésie »: 1070-3. 
confrères traducteurs, n'hésite pas à affirmer que, dans l'œuvre complète d'un poète, une traduction peut constituer un chef d'œuvre supérieur à une création proprement dite :

Plus récemment, dans la traduction du Paradis perdu, ouvrage tantôt sublime et tantôt bizarre du génie non moins étonnant que Shakespeare, on a souvent retrouvé tout le talent de M. Delille. (vii)

Marie Joseph Chénier dessine en outre, toujours dans la même préface du Cimetière de campagne, pour les traducteurs contemporains et pour les traducteurs de 1 ;avenir les contours d'un ample corpus poétique de langue anglaise : autres œuvres de Thomas Gray (Hymne à l'adversité, Odes pindariques, Ode sur le collège d'Eton et une "douzaine de fables"), œuvres de John Dryden (Ode sur la musique), de Matthew Prior (Emma), de Thomas Parnell (l'Hermite), de Joseph Addison (l'Epitre sur l'Italie), d'Oliver Goldsmith (le Voyageur et le Village abandonné). Si Gray et Goldsmith, auteurs déjà traduits en français, appartiennent au dernier tiers du XVIIIe siècle et correspondent à une orientation esthétique typique de la fin du XVIIIe siècle, les autres auteurs de la liste se situent plutôt à la charnière entre le XVIIe et le XVIIIe siècle et figurent dans l'anthologie en huit volume de l'abbé Antoine Yart, Idée de la poésie anglaise, ou Traduction des meilleurs poètes anglais (1749-1756). Cet ouvrage, même s'il fut critiqué pour la qualité de ses traductions, «constitue un tournant dans la réception de la poésie anglaise en France » (Lautel-Ribstein 1050). En rassemblant ce corpus linguistiquement ciblé et sélectionné en vue de « versificateurs habiles » (Le cimetière v), Marie Joseph Chénier prend ainsi la posture d'un traducteur d'avant-garde, pourvu d'un éthos critique. Sa propre traduction de Gray est nouvelle et bilingue : il se présente alors comme un médiateur éclairé et en quelque sorte pilote ouvrant la voie d'une éthique de la traduction à venir. Mais en même temps il s'affirme tout comme d'ailleurs dans l'ensemble de son activité de critique littéraire continuateur et amplificateur d'une dynamique de traduction initiée par Voltaire présenté dans sa préface comme découvreur de la littérature anglaise :

Voltaire, à son retour de Londres, où l'avaient contraint à se réfugier les premières persécutions qu'il eut essuyées en France, fit connaître à sa patrie la philosophie et la littérature des Anglais. Il puisa dans leurs poètes des beautés fortes qu'il sut encore embellir. (iv)

La représentation que le très voltairien Marie Joseph Chénier développe de l'histoire littéraire est ainsi résolument progressive et optimiste, à l'inverse de celle que promeut La Harpe dans son Cours général de littérature ou Lycée : la vision du XVIIIe siècle y est absolument négative, les Lumières n'étant pour La Harpe que 'philosophisme' dégradateur, introducteur d'une révolution foncièrement barbare. ${ }^{4}$

Une phrase à la teneur implicite forte, située dans le texte à l'articulation de sa brève rétrospective historique et du " programme " souhaitable des traductions à venir, attire l'œil : «Quand il devient difficile d'oser penser soi-

\footnotetext{
${ }^{4}$ Le terme 'philosophisme' est ainsi constamment employé par La Harpe et sera repris par Chateaubriand, avec de nombreuses occurrences, dans les Mémoires d'outre-tombe.
} 
même, on peut encore traduire. » (v) Le ton sentencieux semble indiquer qu'à ce moment, la traduction n'est plus pour Chénier l'homme de lettres une pratique littéraire parmi d'autres. Déjà auteur d'un pamphlet intitulé De la liberté du théâtre en France publié en 1789, dans lequel il défendait sa pièce Charles $I X$, Chénier n'a semble-t-il plus la même latitude. En effet, Adolphe Lieby voit dans cette remarque les conséquences de l'interdiction de jouer sur scène sa pièce Philippe II, sujet espagnol qui avait déjà été censuré dans la période prérévolutionnaire : la pièce dépeignant la mort de Don Carlos, fils du roi Philippe II, ne participait pas au maintien des relations franco-espagnoles (Lieby 202-204, 220, 436). Par ce commentaire, Marie Joseph Chénier suggère que, sous l'empire, la pratique de la traduction peut constituer un refuge en s'inscrivant dans une dynamique de compensation d'une liberté de pensée et d'expression compromise par la censure impériale dont on sait qu'elle fut encore plus dure et plus efficace que toutes les censures antérieures. Ce faisant il semble vouloir se rapprocher de la figure de Voltaire persécuté mais aussi traducteur-découvreur, figure qu'il évoque (ou invoque) à deux reprises, la seconde pour rappeler que ce " grandhomme » est " aujourd'hui si ridiculement harcelé » (Le cimetière iv). Faudrait-il y lire la revendication d'une certaine filiation ? Subissant le poids de contraintes idéologiques, banni du système officiel de production, l'homme de lettres peut à nouveau s'exprimer et rejoindre le système dont il est exclu par le biais de la traduction. C'est en effet à cette période que Chénier va travailler sur une traduction de Sophocle et aux adaptations de Nathan le sage de Lessing et de l'Ecole de la médisance de Sheridan (Lieby 427, 436).

Enfin - on est en 1805, moment de la bataille d'Austerlitz et de la préparation de l'invasion de l'Angleterre- Marie Joseph Chénier inscrit la pratique de la traduction littéraire au cœur d'une politique européenne dominée par le principe d'une recherche de la paix entre les Etats :

Les littératures ne sont jamais en guerre. Il peut exister des querelles politiques entre les gouvernements; le vœu philanthropique de Sully, de l'abbé de SaintPierre et de J.J.Rousseau peut n'être encore que le rêve des hommes de bien : mais il existe pour le génie un traité de paix perpétuelle qui doit être religieusement observé. (Le cimetière ix)

En se référant notamment au Projet pour rendre la paix perpétuelle en Europe de l'abbé de Saint-Pierre, Marie Joseph Chénier refuse hautement que les tensions entre les Etats se répercutent sur les échanges littéraires entre les nations et n'hypothèquent ainsi l'activité de traduction. Cette revendication d'un pacifisme hérité des Lumières s'oppose évidemment à la politique belliciste de l'empire et signale la particularité de la position du traducteur, défenseur d'une médiation interculturelle mais néanmoins exposé aux contraintes du système idéologique dans lequel il évolue.

Mais avant de publier cette traduction du Cimetière de campagne de Gray, Marie Joseph Chénier a signalé dans une intervention publique précise, que l'activité de traduction avait une dimension éminemment politique et sociale et méritait la reconnaissance officielle de la nation, prolongeant en 
quelque sorte la pétition qu'il avait soumise à l'Assemblée sur la naturalisation française d'écrivains étrangers (Bingham 1966). Dans son Rapport au nom du comité d'instruction publique sur des secours à accorder aux savants et aux artistes (séance de l'Assemblée nationale du 14 nivôse an III, 3 janvier 1795), Marie Joseph Chénier propose de partager la somme allouée de 300000 livres entre gens de lettres, artistes et savants méritants mais vivant dans le besoin. Chénier est très clair sur l'apport fondamental de ces hommes éclairés à la société tout comme sur la responsabilité de la société envers eux : «... on sert son pays en l'éclairant : les hommes qui font faire un pas à la raison publique sont aussi les défenseurs de la patrie » (380) et " les arts sont une propriété nationale; les encouragements qu'ils réclament sont une dette publique » (382). Parmi les cent seize personnalités nommées et destinées à se partager la dotation, il est dix traducteurs explicitement désignés comme tels :

Bitaubé, traducteur d'Homère ... Delille, auteur de la traduction en vers des Géorgiques ... Dutheil, traducteur d'Eschyle ... Larcher, traducteur d'Hérodote ... Blavet, traducteur de Smith ... Dureau de la Malle, traducteur de Tacite ... Saint-Ange, auteur de la traduction en vers des Métamorphoses d'Ovide ... Mandar Théophile, traducteur de plusieurs ouvrages anglais ... Deshautesrayes, traducteur d'ouvrages orientaux ... Griffet, traducteur de plusieurs ouvrages anglais. (Rapport au nom 383-5)

La figure du traducteur acquiert donc une nouvelle facette, celle d'homme public représentant une classe d'homme de lettres encore peu valorisée même si sous la Ière République « ... on commençait alors à prendre au sérieux la professionnalisation de ce corps de métier, qui n'avait cessé de s'affirmer depuis deux siècles » (Nies et Tran-Gervat 113)..$^{5}$ Chénier ne représente cependant pas tous les traducteurs : la liste est le résultat d'une sélection. D'ailleurs, loin cette fois de toute tonalité élogieuse, Marie Joseph Chénier, dont la propension à la polémique était notoire, n'hésite pas à dénoncer ses adversaires idéologiques en tant que piètres traducteurs :

Epigramme sur les trois traducteurs en vers de la Jérusalem délivrée.

Clément, La Harpe et Lormian-Balour,

Vont traduisant le chantre d'Herminie.

Ainsi traduire est pure calomnie :

Leurs vers menteurs l'ont rendu sec et lourd.

Qu'eût fait Pâris entre ces trois grands hommes?

Eût-il donné la pomme à l'un des trois ? Non : mais, entre eux ne pouvant faire un choix,

A tous les trois il eût jeté des pommes. (Euvres posthumes II, 349)

Mais c'est sans doute dans son Tableau historique de l'état et des progrès de la littérature française depuis 1789 que Marie Joseph Chénier manifeste le plus clairement l'importance qu'il accorde à la traduction et sa volonté de l'inclure dans le canon littéraire qu'il définit et cherche à promouvoir

\footnotetext{
${ }^{5}$ Ce document et cette décision devancent le projet de création d'un établissement public finançant les traductions évoqué dans un débat entre Volney et le citoyen Latapie à l'Ecole normale. (Nies et Tran-Gervat 112-3).
} 
contre vents et marées : contre le rejet des Lumières et de la Révolution par La Harpe, contre la critique du 'philosophisme' et de la Révolution par Chateaubriand. Le projet de Marie Joseph Chénier est énoncé clairement dès l'introduction du Tableau :

La France agrandie n'est pas devenue stérile en talents. Nous rassemblerons sous les yeux des Français les éléments actuels de cette littérature française, dont une envieuse ignorance dénigrait, à chaque époque, et les chefs d'œuvre et les classiques, mais qui fut toujours honorable, et qui même aujourd'hui, malgré des pertes nombreuses, demeure encore, à tous égards, la première littérature de l'Europe. (Tableau 26)

Dans ce canon littéraire incluant la production littéraire et philosophique du siècle des Lumières et des années révolutionnaires, l'accroissement du nombre et de la qualité des ouvrages traduits est présenté à la fois comme le meilleur garant de l'appui sur la tradition classique et comme le révélateur de l'agrandissement géopolitique contemporain de la France par les conquêtes révolutionnaires et impériales :

Parmi les imitateurs, M. Parseval de Grandmaison, à qui l'on doit les Amours épiques, et M. Luce de Lancival, auteur d'Achille à Scyros, doivent être distingués de la foule : mais des traductions du premier mérite nous occuperont bien davantage. Virgile et Milton semblent parler eux-mêmes notre langue; et grâce à un classique vivant, que ce mot fera nommer, grâce encore à $\mathrm{M}$. de Saint-Ange, habile et laborieux traducteur d'Ovide, nous aurons le plaisir d'observer qu'à cet égard l'époque actuelle est supérieure à toute autre. On n'avait pas porté si loin jusqu'à ce jour, au moins en des ouvrages d'une telle importance, l'art difficile de conquérir les beautés de la poésie étrangère, et de traduire le génie par le talent. (19-20)

Dans le Tableau, les nouvelles traductions des œuvres antiques sont signalées et l'objet d'amples et parfois sévères commentaires. Le chapitre VII consacré à la Poésie épique fait une place explicite (signalée même dans la table des matières) aux récentes « imitations et traductions en vers ». Pour Marie Joseph Chénier, les vingt dernières années sont une période exceptionnellement riche pour ce qui est des traductions des grandes épopées anciennes et modernes même s'il note toutefois qu'Ossian n'égalera jamais Homère (293). C'est dans cette section que, commentant les mérites de Delille, traducteur de l'Enéide et du Paradis perdu de Milton, Chénier développe une brève théorie de la traduction :

... ces deux ouvrages soutiennent avec honneur la renommée de M. Delille. Que d'autres lui reprochent d'avoir négligé tel mot, d'avoir modifié telle image, qu'ils veuillent lui enseigner le latin, l'anglais et le ramener impérieusement à la traduction littérale, système vicieux en prose et ridicule en vers, nous ne suivrons pas leur exemple. Copier servilement des formes étrangères, c'est travestir à la fois sa propre langue et l'auteur que l'on interprète; ce n'est pas traduire c'est calomnier. Voulez-vous faire un portrait ressemblant? Saisissez la physionomie. Voulez-vous rendre fidèlement un classique, en conservant toutes ses pensées ? Ecrivez, s'il est possible, comme il eût écrit en votre langue ; car ce n'est point le mot, c'est le génie qu'il faut traduire. (298-9) 
Chénier reprend donc l'idée de la primauté du génie de l'auteur sur la langue d'arrivée, telle que Delille l'avait explicitée dans le « Discours préliminaire » à sa traduction des Géorgiques (Tran-Gervat et Weiman 310-4 ; D’hulst 119-25). Toutefois les mots de Chénier feront date puisqu'ils seront repris comme principes de toute démarche traductive par Bernard Jullien, auteur d'une Histoire de la poésie française à l'époque impériale (1844) et directeur de la Revue de l'instruction publique, créée en 1842 par Louis Hachette (Weinmann 52). Le sixième chapitre consacré aux romans souligne lui aussi l'importance des traductions anciennes (du Don Quichotte notamment) et des traductions plus récentes. Marie Joseph Chénier note ainsi à propos de la nouvelle traduction du Don Quichotte par M. du Bournial :

M. du Bournial ... est simple et n'est point trivial; il est surtout copiste fidèle; il l'est au point, qu'en plaçant le français à côté de l'espagnol, vous reconnaissez dans la plupart des phrases, la même marche, les mêmes constructions, les mêmes tours ... (266-7)

Mais Marie Joseph Chénier insiste plus particulièrement sur l'importance quantitative des romans anglais traduits en masse pour noter que « ... trois ans ne suffiraient pas pour les lire » (275), et sur la qualité du Werther de Goethe, alors que le roman de Goethe intitulé Alfred est par contre vivement critiqué. Mais globalement Marie Joseph Chénier est nettement sceptique à l'égard du genre romanesque ${ }^{6}$ et des romans traduits qui, selon lui, corrompent la langue en y introduisant « des tours anglais ou germaniques » (278).

Mais nous percevons là les limites de l'ouverture du canon littéraire élaboré par Marie Joseph Chénier en matière de traduction : Chénier est et restera hostile aux textes, traduits ou non, susceptibles de bouleverser les principes très classiques de son esthétique. Et dans les traductions qu'il cite et commente, les traductions des textes antiques sont nettement majoritaires : homme des Lumières et révolutionnaire, Marie Joseph Chénier se veut à la fois le gardien vigilant de la tradition littéraire classique et un critique ouvert aux productions étrangères à condition qu'elles ne rompent ou n'incitent pas à rompre avec l'esthétique classique.

6 « En France, en Angleterre, en Allemagne, il existe pour les romans des manufactures établies ...» $(275)$. 


\section{Bibliographie}

Baczko, Bronislav. Comment sortir de la Terreur. Thermidor et la Révolution. Paris : NRF, 1989.

Bingham, Alfred Jepson. Marie Joseph Chénier: Early Political Life and Ideas (1789-1794). New York: Privately printed, 1939.

---. « Marie Joseph Chénier and French Culture during the French Revolution. » The Modern Language Review 61. 4 (1966) : 593-600.

Castel de Saint-Pierre, Charles-Iréné. Projet pour rendre la paix perpétuelle en Europe. Utrecht : A. Schouten, 1713.

Chateaubriand, François-René (de). Mémoires d'outre-tombe. Paris : Gallimard, 1997.

Chénier, Marie Joseph. Caüus Gracchus, Tibère : deux tragédies politiques. Ed. Pierre Frantz et François Jacob. Paris : Chrystel, 1998.

--. Le Cimetière de campagne. Elégie anglaise de Gray, Traduction nouvelle en vers français. Paris : Dabin, an XIII, 1805.

---. De la liberté du théâtre en France. (s.l.), (s.n.) 1789 (Gallica ark:/12148/bpt6k57015q) [consulté en mai 2015]

---. Euvres choisies. Paris : Baudouin-Frères, 1821, 4 vol.

---. Euvres posthumes. 3 vols. Paris : Guillaume, 1825.

---. Poésies lyriques de Marie Joseph Chénier. Paris : Didot l'aîné, an V de la

République.

--. Rapport au nom du comité d'instruction publique sur des secours à accorder aux savants et aux artistes (14 nivôse an III) dans Procès-verbaux du Comité d'instruction publique de la Convention nationale, publ. et annotés par M. J. Guillaume. Paris : Imprimerie nationale, 380-386. (Gallica, cote NUM : 29292) [consulté en mai 2015]

---. Tableau historique de l'état et des progrès de la littérature française depuis 1789. Euvres posthumes. vol. III. Paris : Guillaume, 1825.

---. Théâtre de M. -J. de Chénier. 2 vols. Paris : Baudouin Frères, 1821.

Domine, Jean-François. " Le chant du départ de Marie Joseph Chénier et Etienne Méhul. » Annales historiques de la Révolution française 329 (juillet-septembre 2002) : 89-100.

D'hulst, Lieven. Cent ans de théorie française de la traduction. De Batteux à Littré (1748-1847). Presses universitaires de Lille, 1990.

Ferry, Ariane et Humbert-Mougin, Sylvie. «Théâtre ». Histoire des traductions en langue française. XIXe siècle. Dir. Yves Chevrel, Lieven D'hulst, Christine Lombez. Paris : Verdier, 2012. 443-535.

Lautel-Ribstein, Florence. "Poésie ». Histoire des traductions en langue française. XVIIe-XVIIIe siècles. Dir. Yves Chevrel, Annie Cointre, YenMaï Tran-Gervat. Paris : Verdier, 2014. 949-1120.

Lechevalier, Claire et Marie, Laurence. « Théâtre ». Histoire des traductions en langue française. XVIIe-XVIIIe siècles. Dir. Yves Chevrel, Annie Cointre, Yen-Maï Tran-Gervat. Paris : Verdier, 2014. 855-941.

Lieby Adolphe. Etude sur le théâtre de Marie Joseph Chénier. Thèse pour le doctorat présentée à la Faculté des Lettres de l'Université de Paris. Paris : Société française d'imprimerie et de librairie, 1901.

Nies, Fritz et Tran-Gervat, Yen-Maï. « Traducteurs ». Histoire des traductions 
en langue française. XVIIe-XVIIIe siècles. Dir. Yves Chevrel, Annie Cointre, Yen-Maï Tran-Gervat. Paris : Verdier, 2014. 103-85.

Pertué, Michel et Soboul, Albert. Dictionnaire historique de la Révolution française. Paris : Presses Universitaires de France, coll. Quadrige, 2005, "Chénier Marie Joseph." 216-7.

Tatin-Gourier, Jean-Jacques. Procès du 'philosophisme révolutionnaire' et retour des Lumières des lendemains de Thermidor à la Restauration. Presses universitaires Laval, 2008.

Tran-Gervat, Yen-Maï et Weinmann, Frédéric. « Discours. » Histoire des traductions en langue française. XVIIe-XVIIIe siècles. Dir. Yves Chevrel, Annie Cointre, Yen-Maï Tran-Gervat. Paris: Verdier, 2014. 249-367.

Walton, Charles. " Charles IX and the French revolution: Law, vengeance, and the revolutionary uses of History. \ European Review of History/Revue européenne d'histoire 4-2, (1997), 127-146. Web. 31 mai 2008. DOI: 10.1080/13507489708568182 [consulté en mai 2015]

Weinmann, Frédéric. « Théories. » Histoire des traductions en langue française. XIXe siècle. Dir. Yves Chevrel, Lieven D'hulst, Christine Lombez. Paris : Verdier, 2012. 51-148. 